\title{
Tumor Progression
}

National Cancer Institute

\section{Source}

National Cancer Institute. Tumor Progression. NCI Thesaurus. Code C17609.

A pathologic process in which alterations at the molecular level result in a more aggressive cytologic and phenotypic profile and clinical course of a neoplasm. 